\title{
COVID-19 and Its Relation to Takotsubo Cardiomyopathy
}

\author{
Wedad Fisal Khader ${ }^{1}$, Mohammed AlBashtawy ${ }^{2}$, Jamal Qaddumi ${ }^{3}$, Sa'd Albashtawy ${ }^{4}$, Fadi Fawares ${ }^{5}$, \\ Zaid AlBashtawy ${ }^{6}$, Abdullah Alkhawaldeh ${ }^{7}$ \\ ${ }^{1}$ Master Student, Critical Care Nursing, ${ }^{2}$ Professor, Princess Salma Faculty of Nursing, AL al-Bayt University, \\ Mafraq, Jordan, ${ }^{3}$ Associate Professor, Faculty of Medicine and Health Sciences, An-Najah National University, \\ Nablus-Palestine, ${ }^{4}$ Faculty of Medicine, Hashemite University, Zarqa, Jordan, Jordan, ${ }^{5}$ Nurse Educator at King \\ Hussein Cancer Center, Amman, Jordan, ${ }^{6}$ Faculty of Medicine, Yarmouk University, Irbid, Jordan, ${ }^{7}$ Assistance \\ Professor, Princess Salma Faculty of Nursing, AL al-Bayt University, Mafraq, Jordan
}

\begin{abstract}
Takotsubo Cardiomyopathy (TC) is a stress caused heart condition, it might be transient or complicated with other irreversible conditions. Recently, many case reports were released indicating of COVID-19 cases complicated with TC. This paper aimd to review studies that examined the correlation between COVID-19 and TC. An electronic search was carried out using PubMed and Google Scholar data base.The TC is one of the major COVID-19 complications that must be taken into consideration. The exact mechanism is still unknown. Further studies are needed in order to confirm this relationship in addition to investigating the underlying mechanism.
\end{abstract}

Keywords: Takotsubo cardiomyopathy, Takotsubo syndrome, COVID-19.

\section{Introduction}

Takotsubo Cardiomyopathy (TC) is a cardiac event that is induced by stress, and it's initial presentation is similar to that of Myocardial Infarction (MI) ${ }^{(1)}$. The TC is usually caused by major stressful or emotional events; it was also found that TC induce systemic inflammatory state that remains for more than 5 months after the initial presentation $^{(2)}$.

Patients with TC could have normal ECG, STelevation, and ST/T wave changes or transient left bundle branch block or arrythmias ${ }^{(3)}$. There is no clear optimal treatment of TC, however, in some cases the infusion of inotropic agents could be a good treatment option ${ }^{(4)}$.

In March 11, 2020, World Health Organization (WHO) announced COVID-19 outbreak as a global

\section{Corresponding Author:}

Abdullah Alkhawaldeh,

$\mathrm{PhD}$, Assistance Professor, Princess Salma Faculty of

Nursing, AL al-Bayt University, Mafraq, Jordan.

Email: dr-abd@aabu.edu.jo, abdo_752012@yahoo.com pandemic. Hence, it was of a high importance to do comprehensive studies regarding the disease and its possible outcomes. Since then, several reports were released shed the light on the link between COVID-19 and $\mathrm{TC}^{(5,6,7)}$.

This paper aimd to review studies that examined the correlation between COVID-19 and TC.

\section{Methods}

Search methods: The electronic searching was conducted in PubMed and Google Scholar. Key search terms used: Takotsubo cardiomyopathy, Takotsubo syndrome, COVID-19.

The studies that focused on the topic of Takotsubo cardiomyopathy and COVID-19, were published in English between 2016 and 2020 were included in the review. While, studies published before 2016 were excluded.

Search outcome: Exploring of literature yield about 35 studies for review, after reading the studies about 25 study were excluded and 10 studies met the inclusion 
criteria.

\section{Discussion}

As a result of the conducted search, several studies were carried out that explored the correlate between COVID-19 and TC. According to (6), they studied the characteristics of patients who had confirmed COVID-19 and TC based on transthoracic echo cardiographic features, accordingly, the authors suggested that the TC was caused by the COVID-19 associated emotional distress.

A case report of a 67 years old female patient, who was presented complaining of fever and several other symptoms and was diagnosed as COVID-19 case, it was noted that the patient was anxious, three days later she developed TC with atrial fibrillation, she was treated with dual anti-platelet therapy and was discharged with caution ${ }^{(7)}$.

Another case report was released about a 40 years old male patient who was admitted to emergency room complaining of cough and chest pain, later on he was diagnosed with TC and he was treated by rescue TPE for 5 days, the authors could not exclude other cardiac causes of TC due to the sensitivity of dealing with COVID-19 patients. However, they suggested that it was caused by COVID-19 emotional stress ${ }^{(5)}$

A study reported a case of TC and COVID-19 in 52 years old male patient, he was admitted complaining of shortness of breath. The lab tests revealed elevated CRP and D-Dimer. He was treated by colchicine and methylprednisolone and heparin continuous infusion. It was suggested that the cytokine storm caused by COVID-19 induced the TC along with the emotional distress ${ }^{(8)}$.

On the other hand, some other studies reported that patients were diagnosed with TC without the presence of COVID-19, but the underlying cause was referred to COVID-19. In Greece, a case report indicated that a patient was admitted complaining of chest pain after she was watching the numbers of COVID-19 deaths, ECG showed ST elevation and impaired left ventricular systolic function and was diagnosed with $\mathrm{TC}^{(9)}$. In USA, a case report indicated that a 72 years old female was presented and diagnosed with COVID-19 and ischemic stroke, incidentally, she was also diagnosed with TC which was complicated with cardiogenic shock and leaded to death ${ }^{(10)}$.

In another case report, a 72 years old female patient was diagnosed with COVID-19, seven days later she developed TC. The report recommended that carrying frequent ECG for COVID-19 patients due to the high risk of developing TC. Moreover, the report stated that the QTc prolongation of TC could be further complicated by the medications that cause QTc prolongation leading to lifethreatening arrhythmias, hence, caution must be taken while dealing with such cases ${ }^{(11)}$.

One possible explanation that the cortisol very high levels in concert with catecholamines high levels related to the common basic mediator(s) (cytokine storm and in-flammation) might produce a direct 'toxic' impact oncardiomyocytes in COVID-19 patient, and have a role in the occurrence of TCM ${ }^{(12)}$. Another possible mechanism was suggested that TC might be caused by the viral entry via ACE-2 which is highly expressed in lung and cardiac tissue ${ }^{(13)}$.

Finally, to increase our understanding about the TC pathophysiology and the role of stress-related hormones. There is a needed for studies that compare stress-related hormones (such as the cortisol levels) among patients with TC and COVID-19 and others with COVID-19 without TCM ${ }^{(12)}$. Also, in the period of COVID-19, health care providers should give attention to different cardiovascular conditions associated with COVID-19 (14 ), TCM is one of these conditions that can be activated by physical and emotional impact of COVID-19 ${ }^{(15-27)}$.

\section{Conclusion}

The recent studies suggest that the $\mathrm{TC}$ is one of the major COVID-19 complications that must be taken into consideration. Many mechanisms where suggested including the direct emotional distress and psychological stress, the elevated levels of cortisol and other stress hormones, the cytokine storm and the entry of the virus via ACE-2. However, the real underlying mechanism is still unknown. Further studies are needed in order to confirm the relationship between COVID-19 and TC. Moreover, healthcare providers have to do frequent ECG for COVID-19 patients in order to detect any cardiac complications including TC. 
Conflict of Interest: No conflict of interest.

Ethical Clearance: Taken from Princess Salma Faculty of Nursing, AL al-Bayt University ethical committee.

\section{Source of Funding: Self.}

\section{References}

1. Scally, C., Rudd, A., Mezincescu, A., Wilson, H., Srivanasan, J., Horgan, G., ... Dawson, D. K. Persistent Long-Term Structural, Functional, and Metabolic Changes After Stress-Induced (Takotsubo) Cardiomyopathy. Circulation 2017; $1-17$.

2. Scally, C., Abbas, H., Ahearn, T., \& Srinivasan, J. Myocardial and Systemic Inflammation in Acute Stress-Induced ( Takotsubo ) Cardiomyopathy. circulation 2018; 1-31.

3. Dawson, D. K. Acute stress-induced (Takotsubo) cardiomyopathy. Heart 2017; 0:1-7.

4. Mrozek, S., Srairi, M., Marhar, F., Delmas, C., Gaussiat, F., Abaziou, T., ... Geeraerts, T. Successful treatment of inverted Takotsubo cardiomyopathy after severe traumatic brain injury with milrinone after dobutamine failure. Heart and Lung The Journal of Acute and Critical Care 2016; 45:406-408.

5. Faqihi, F., Alharthy, A., Alshaya, R., Papanikolaou, J., Kutsogiannis, D. J., Brindley, P. G., \& Karakitsos, D. Reverse takotsubo cardiomyopathy in fulminant COVID-19 associated with cytokine release syndrome and resolution following therapeutic plasma exchange : a case-report. BMC Cardiovascular Disorders 2020; 0:1-7.

6. Giustino, G., Croft, L. B., Oates, C. P., Rahman, K., Lerakis, S., Reddy, V. Y., \& Goldman, M. Takotsubo Cardiomyopathy in COVID-19. Journal of the American College of Cardiology 2020; 76:628-629.

7. Sattar, Y., Connerney, M., Ullah, W., Philippou, A., Slack, D., McCarthy, B., ... Alraies, M. C. COVID-19 Presenting as Takotsubo Cardiomyopathy Complicated with Atrial Fibrillation. IJC Heart\&VasculatureJournal 2020; 29:1-4.
Medico-legal Update, July-September 2021, Vol.21, No. $3 \mathbf{2 6 5}$

8. Taza, F., Zulty, M., Kanwal, A., \& Grove, D. Takotsubo cardiomyopathy triggered by SARS- infection in a critically ill patient. BMJ 2020; 1314.

9. Giannitsi, S., Tsinivizov, P., Poulimenos, L. E., Kallistratos, M. S., Varvarousis, D., \& Manolis, A. J. Stress induced ( Takotsubo ) cardiomyopathy triggered by the COVID - 19 pandemic. Experimental and Therapeutic Medicine 2020; 20:2812-2814.

10. Kariyanna, PT., et al. "Apical Takotsubo cardiomyopathy in a COVID-19 patient presenting with stroke: a case report and pathophysiologic insights." American journal of medical case reports 2020, 8:350-357.'

11. Osch, D. Van, Asselbergs, F. W., \& Teske, A. J. Takotsubo cardiomyopathy in COVID-19: a case report. Haemodynamic and therapeutic considerations. European Heart Journal 2020; 0-5.

12. Salah, Husam M., and Jawahar L. Mehta. "Takotsubo cardiomyopathy and COVID-19 infection." European Heart Journal-Cardiovascular Imaging (2020).

13. Tsao, C. W., Strom, J. B., Chang, J. D., \& Manning, W. J. COVID-19-Associated Stress (Takotsubo) Cardiomyopathy. Circulation: Cardiovascular Imaging 2020; 1-4.

14. Aloush , S., Abdelkader , F., Al-Sayaghi , K., Tawalbeh, L., Suliman, M., ALBashtawy, M. and Shaban,.1. Compliance of Nurses and Hospitals with Ventilator Associated Pneumonia Prevention Guidelines: A Middle Eastern Survey. Journal of Nursing Care Quality 2017.

15. Okura, H. Update of takotsubo syndrome in the era of COVID-19. Journal of Cardiology 2020; 1-9.

16. Tawalbeh, L., Tubaishat, A. , Batiha, A., AlAzzam, M. \& ALBashtawy, M. .The Relationship between Social Support and Adherence to Healthy Lifestyle among Patients with Coronary Artery Disease in the North of Jordan. Clinical Nursing Research 2015; 24:121-38.

17. Batiha, AM. AlAzzam, M. ALBashtawy,M., Tawalbeh , L., Tubaishat, A., Alhalaiqa, F. The Relationship between Hypertension and Anthropometric Indices in a Jordanian Population. 
Advanced studies in biology 2015; 7:233-243.

18. Sulaiman, M. , \& Aljezawi, M., ALBashtawy, M., Fitzpatrick, J., Aloush . S. \& Al-Awamreh, k Exploring Safety Culture in Jordanian Hospitals: A Baseline Study". Journal of Nursing Care Quality 2017; 32:p E1-E7.

19. Qaddumi, J., Holm,M., Alkhawaldeh, A., ALBashtawy, M., AL Omari,O., Batiha,AM., Alhalaiqa,F., Suliman,M., Aljohani, K. \& Da'she, A. Prevalence of Hypertension and Prehypertension among Secondary School Students. International Journal of Advanced Nursing Studies 2016; 5:240-245.

20. Howeri, N., AlBashtawy, M., Ozaybi, N., Alkhawaldeh, A., Al Azzam, M., Albashtawy, S., Khatatbeh, M., Khraisat, O., AlBashtawy Z., and Suliman M. Nurses' Knowledge Regarding Care Provided to Patients with Angina. EC Pulmonary and Respiratory Medicine 2020; 10:01-04.

21. Suliman, M,. Aloush, S,. Aljezawi, M. \& ALBashtawy, M. Knowledge and practices of isolation precautions among nurses in Jordan. American Journal of Infection Control 2017.

22. Omari, O., Alkhawaldeh, A., ALBashtawy, M., Qaddu,J, Holm, M., \& ALOmari, D. A Review of the Short Form Health Survey - Version 2. Journal of Nursing Measurement 2019; 27:76-85.
23. Aloush, S.M., Al-Sayaghi, K., Tubaishat, A., Dolansky, M., Abdelkader, F.A., Suliman, M., ALBashtawy, M., Halabi, M. Compliance of Middle Eastern hospitals with the central line associated bloodstream infection prevention guidelines. Applied Nursing Research 2018; 43:5660.

24. Tawalbeh, L.I., Al-Smadi, A.M., AlBashtawy, M., AlJezawi, M., Jarrah, M., Al-Mahasees, A., Aloush, S. The Most and the Least Performed Self-Care Behaviors Among Patients with Heart Failure in Jordan, Clinical Nursing Research 2020; 29:108-116

25. Khraisat, O. Al-awamreh, K\& AlBashtawy M. Shared Governance: A children's hospital journey to clinical nursing excellence" Journal of Research in Nursing 2020.

26. Al Kazaleh A., and AL Bashtawy M. "Therapeutic Communication Skills in Nursing Education and Practice". EC Psychology and Psychiatry 2019; 8:01-04

27. AL-rawashdeh, N., Al Bashtawy, M., Ozaybi, N., Alkhawaldeh, A., Albashtawy, B., Albashtawy, S., Khatatbeh, M., Khraisat, O., AlBashtawy Z., and Suliman M.. Nurses Roles in Providing Care for Patient with COVID-19. EC Emergency Medicine and Critical Care 2020; 5. 